\title{
Demonstration of the parallel axis theorem through a smartphone
}

I. Salinas, M. H. Gimenez, J. A. Monsoriu, and J. A. Sans

Citation: The Physics Teacher 57, 340 (2019); doi: 10.1119/1.5098929

View online: https://doi.org/10.1119/1.5098929

View Table of Contents: https://aapt.scitation.org/toc/pte/57/5

Published by the American Association of Physics Teachers

\section{CAPTURE WHAT'S POSSIBLE}

\section{WITH OUR NEW PUBLISHING ACADEMY RESOURCES}

\section{Learn more $\Theta$}




\section{Demonstration of the parallel axis theorem through a smartphone}

I. Salinas, M. H. Gimenez, J. A. Monsoriu, and J. A.

Sans, Universitat Politècnica de València, València, Spain;

juasant2@upv.es

$\mathrm{N}$ ew learning strategies try to extend the use of common devices among students in physics lab practices. In particular, there is a recent trend to explore the possibilities of using smartphone sensors to describe physics phenomena. ${ }^{1,2}$ On the other hand, the study of the moment of inertia by the use of the torsion pendulum is a typical example in the first courses of fundamentals of physics. ${ }^{3}$ This example allows the exploration of harmonic motion, Newton's second law, the moment of inertia theory, and the parallel axis theorem all in one. Here, we report the use of the accelerometer sensor of a smartphone to visualize and demonstrate the parallel axis theorem in a torsion pendulum.

\section{Experiment}

The study of the torsion pendulum motion will serve to visualize forced, damped, or simple harmonic motion and explore the implications of the moment of inertia and the parallel axis theorem. ${ }^{4,5}$

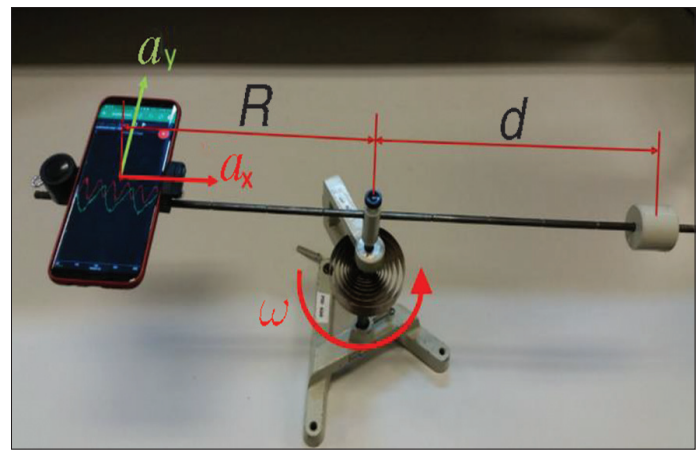

Fig. 1. Scheme of the experiment performed.

In the experiment described in Fig. 1, a rod rotates around its rotation axis subjected to the force of a spring (stiffness $k$ ). In order to measure the acceleration caused in the extreme of the rod, we took advantage of the accelerometer sensor of the smartphone, whereas a known mass $m$ is placed at several distances from the center of the rod. Thus, applying Newton's second law, we know that the moment of force or torque $M_{\mathrm{T}}$ is related to the angular acceleration $\alpha$ and the moment of inertia $I$. Therefore, the motion is dominated by the stiffness of the spring and the angle shifted $\theta$ with the following relation:

$$
M_{\mathrm{T}}=I \alpha=-k \theta .
$$

Then, the equation that must be solved is

$$
\ddot{\theta}+\frac{k}{I} \theta=0,
$$

where the angular frequency is:

$$
\Omega=\sqrt{\frac{k}{I}}=\frac{2 \pi}{T},
$$

The moment of inertia of the system can be described as the addition of the moment of inertia of its components, as follows:

$$
I=I_{\text {rod }}+I_{\text {smartphone }}+I_{\text {mass }} \text {. }
$$

Now, applying the parallel axis (or Huygens-Steiner) theorem to $m$ that says the moment of inertia around any axis $\left(I_{\text {mass }}\right)$ separated a distance $d$ to the center of mass can be obtained from the moment of inertia of a parallel axis passing through the center of mass of the object $\left(I_{\text {mass } 0}\right)$,

$$
I_{\text {mass }}=I_{\text {mass } 0}+m d^{2} \text {. }
$$

Then, the moment of inertia of the system can be expressed as a function of the distance, such as

$$
I=I_{\text {rod }}+I_{\text {smartphone }}+I_{\text {mass } 0}+m d^{2}=I_{0}+m d^{2} .
$$

The period squared of the oscillation can be expressed as

$$
T^{2}=\frac{4 \pi^{2} I}{k}=\frac{4 \pi^{2} I_{0}}{k}+\frac{4 \pi^{2} m}{k} d^{2} .
$$

In summary, there is a direct relation between the period of the oscillation registered in the extreme of the rod and the distance of the mass to its center of gravity.

\section{Analysis and discussion}

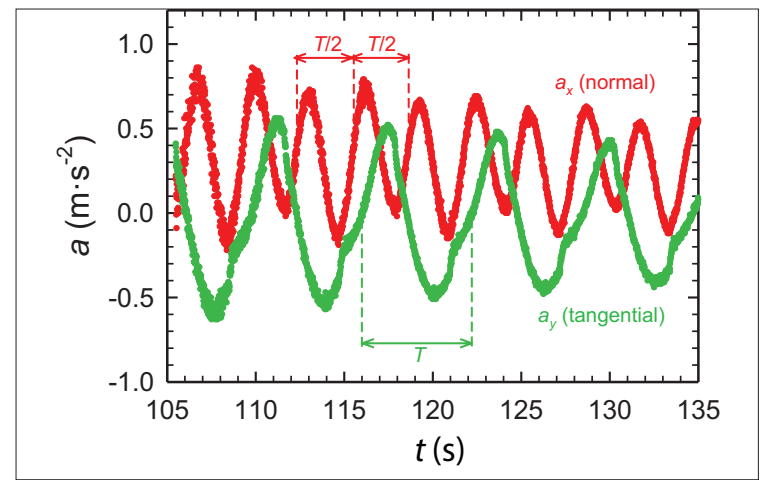

Fig. 2. Plot of the normal and tangential components of the acceleration in function of time placing the mass $m$ at $d=0.20 \mathrm{~m}$.

By means of a slight shift of the rod in the spring, the system starts to oscillate. The oscillations of the system are collected by the Android application Physics Toolbox Suite, ${ }^{6}$ taking advantage of the smartphone's accelerometer sensor. The representation of the oscillations is shown in Fig. 2, which allows us to calculate the period $(T)$ of each oscillation for the normal and tangential component of the acceleration. 
Table I. Average time period of the tangential acceleration for each position (d) of the mass $m$.

\begin{tabular}{|c|c|}
\hline$d(\mathrm{~m})$ & $T_{\text {average }}(\mathrm{s})$ \\
\hline 0.05 & 4.87 \\
\hline 0.10 & 5.18 \\
\hline 0.15 & 5.65 \\
\hline 0.20 & 6.24 \\
\hline 0.25 & 6.92 \\
\hline 0.30 & 7.68 \\
\hline
\end{tabular}

The values of the time period of the tangential component of the acceleration at each position of the mass $m$ of $0.236 \mathrm{~kg}$ are represented in Table I.

The square of the values showed in Table I are displayed in Fig. 3, where one can observe a linear trend, according to Eq. (7). The high quality of the fit $\left(r^{2}=0.9999\right)$ offered a large reliability of the parameters obtained. Thus, from the fit to a linear dependence through minimum squares and comparing with Eq. (7), we can obtain that the slope $a_{\text {slope }}$ is related to the stiffness of the spring as $a_{\text {slope }}=4 \pi^{2} \mathrm{~m} / \mathrm{k}$.

Applying Eq. (1), one can also find the value of the stiffness of the spring by the application of a force at a determined distance. In our case, we have applied $0.25 \mathrm{~N}$ at a distance of $0.3 \mathrm{~m}$ and we have observed that the rod rotated $\pi \mathrm{rad}$, then $k$ $=\left|M_{\mathrm{T}}\right| / \theta=0.0239 \mathrm{Nm}$. The stiffness calculated from Eq. (7) is $k_{\text {measured }}=4 \pi^{2} \mathrm{~m} / \mathrm{a}=0.0231 \mathrm{Nm}$. The discrepancy obtained in the calculation of the stiffness of the spring by both of these methods is less than $4 \%$.

In summary, we present a new way to calculate the stiffness of a spring applying previously acquired knowledge about moment of inertia and parallel axis theorem thanks to the use of the accelerometer sensor of a smartphone. This new method has been proved as an invaluable tool to bring physics experimentation to the students and discover the potential possibilities of this common device as a sensor in a multitude of basic physics experiments.

\section{Acknowledgments}

The authors would like to thank the Institute of Educational Sciences of the Universitat Politècnica de València (Spain) for the support of the Teaching Innovation Groups MoMa and e-MACAFI. JAS acknowledges Ramón y Cajal fellowship program (RYC-2015-17482).

\section{References}

1. M. Monteiro, C. Stari, C. Cabeza, and A. C. Martí, "Magnetic field 'flyby' measurement using a smartphone's magnetometer and accelerometer simultaneously," Phys. Teach. 55, 580 (Dec. 2017).

2. M. H. Giménez, I. Salinas, and J. A. Monsoriu, "Direct visualization of mechanical beats by means of an oscillating smartphone," Phys. Teach. 55, 424 (Oct. 2017).

3. R. Green, "Calibrated torsion pendulum for moment of inertia measurements," Am. J. Phys. 26, 498 (July 1958).

4. J. N. Boyd and P. N. Raychowdhury, "Parallel axis theorem," Phys. Teach. 23, 486 (Nov. 1985).

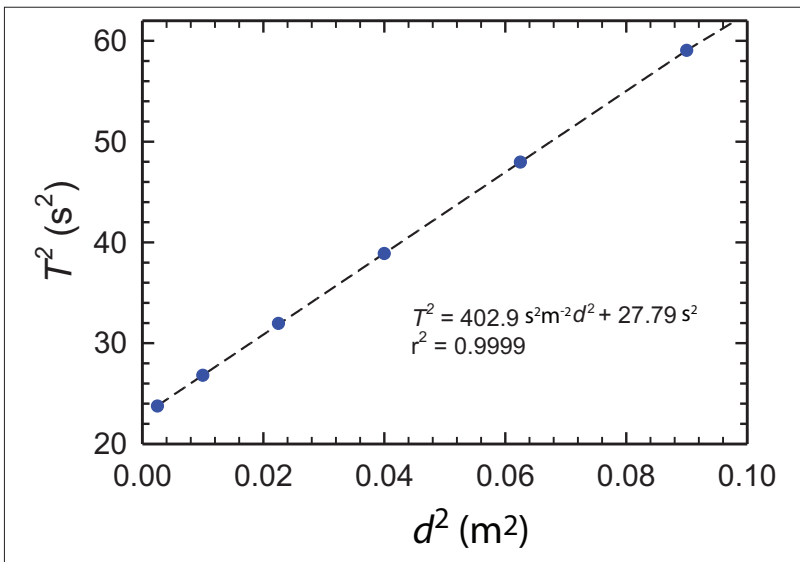

Fig. 3. Linear fit of period squared in function of the square of the distance between the mass and its center of gravity.

5. D. Christie, "Tennis rackets and the parallel axis theorem," Phys. Teach. 52, 208 (April 2014).

6. Physics Toolbox Suite, Google Play, https://play.google.com/ store/apps.

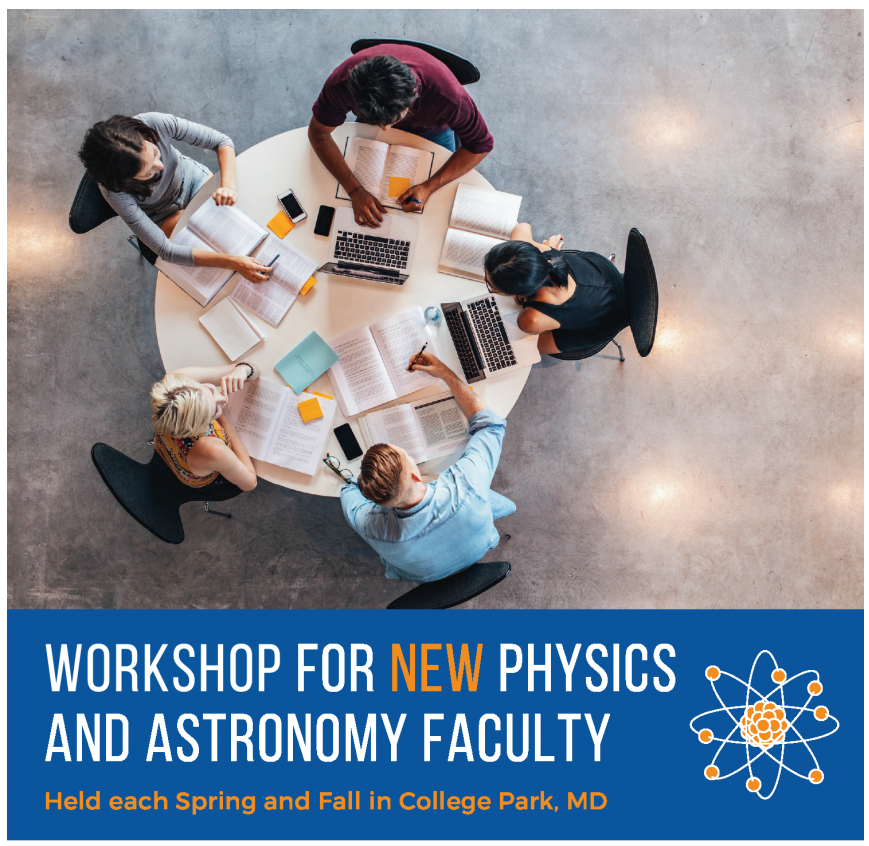

Since 1996, Workshops for New Physics and Astronomy Faculty have introduced faculty members to effective and easy-toimplement interactive engagement teaching methods, grantwriting best practices and plans for preparing for tenure/ promotion decisions. New faculty are nominated by their Department Chair to attend the workshop.

Learn More: Visit aapt.org/conferences/newfaculty Email us: programs@aapt.org or call 301-209-3344 